\title{
Guiding role of seminal tract anatomical study in transurethral seminal vesiculoscopy
}

\author{
Kun Pang ${ }^{1,2,3}$, Wen Yang ${ }^{4,5}$, Jianjun Zhang ${ }^{2,6,7}$, Longjun Cai ${ }^{2,6}$, Bo Chen ${ }^{1}$, Zhiguo Zhang ${ }^{2}$, Lin Hao ${ }^{1}$, Zhenduo Shi ${ }^{1}$, \\ Bo Jiang ${ }^{1}$, Changjie Ouyang ${ }^{8}$, Dewei $\mathbf{Q u}^{8}$, Deguang Wang ${ }^{8}$, Conghui Han ${ }^{1,2,3}$, Wenda Zhang ${ }^{1}$ \\ ${ }^{1}$ Department of Urology, Xuzhou Central Hospital, Xuzhou Clinical College affiliated to Xuzhou Medical University, Xuzhou, China \\ ${ }^{2}$ Department of Urology, The Third Affiliated Hospital of Soochow University, Changzhou, China \\ ${ }^{3}$ Department of Biotechnology, College of Life Sciences, Jiangsu Normal University, Xuzhou, China \\ ${ }^{4}$ Department of Renal Disease, Shandong First Medical University, Tai'an, China \\ ${ }^{5}$ Department of Renal Disease, Affiliated Hospital of Shandong Academy of Medical Sciences, Ji'nan, China \\ ${ }^{6}$ Department of Urology, Suqian People's Hospital of Nanjing Drum-Tower Hospital Group, the Affiliated Suqian Hospital of Xuzhou \\ Medical University, Suqian, China \\ ${ }^{7}$ Department of Urology, Hospital of Xuzhou Medical University, Suqian, China \\ ${ }^{8}$ Department of Anatomy, Xuzhou Medical University, Xuzhou, Jiangsu, China
}

Videosurgery Miniinv 2020; 15 (1): 176-184 DOI: https://doi.org/10.5114/wiitm.2019.86774

\begin{abstract}
Introduction: There have been problems with low qualification operator-related complications and failures of transurethral seminal vesiculoscopy (TSV) in China.

Aim: To study the guiding role of seminal tract anatomical study (STAS) in TSV.

Material and methods: We performed STAS to study the structure, morphology, duct trajectory, and anatomical relationships between the seminal vesicles and the adjacent tissue in pelvic specimens from 12 adult cadavers. Then the surgical effects and complications of 82 cases of TSV performed by 3 doctors were retrospectively studied to compare the difference between the two groups of before and after the anatomical study.

Results: The anatomical studies of the 12 adult cadaveric pelvis specimens identified the lengths and widths of the right- and left-side seminal vesicles and tracts. The TSV can treat lesions located in the distal seminal tract and vesicle, but proximal lesions cannot be reached, which is an anatomical limitation of this technique. There were significant differences in the surgical times and the surgical validity rates between the 2 groups.

Conclusions: Our anatomical study of the seminal tract and seminal vesicles is valuable for guiding TSV in clinical practice.
\end{abstract}

Key words: transurethral seminal vesiculoscopy, ejaculatory duct and seminal vesicle diseases, seminal tract anatomical observation, surgical effect, complications.

\section{Introduction}

Ejaculatory duct and seminal vesicle diseases are common male urological disorders and include chronic seminal vesiculitis, spermatic calculus, sem- inal vesicle cyst, and ejaculatory duct stenosis or obstruction [1]. Seminal tract obstruction may lead to obstructive azoospermia, and affected patients may experience intractable hemospermia, perineal discomfort and pain, ejaculation pain, azoospermia,

\section{Address for correspondence}

Conghui Han and Wenda Zhang, Xuzhou Central Hospital, 199 South Jiefang Road, Xuzhou, 221009, Jiangsu, China,

e-mail: 479920288@qq.com, zhangwd282@163.com 
or infertility [2]. In the past, the outcome of conservative treatment was poor, and open surgery, laparoscopic or even flexible vesiculovasoscopy surgery has been performed for common seminal vesicle diseases [3-8]. For seminal tract and seminal vesicle diseases in certain locations, there is no appropriate and effective treatment method [7]. Transurethral seminal vesiculoscopy (TSV) is an endoscopic technique that has developed rapidly in recent years. Because it is a relatively simple procedure and does not change the normal anatomical structures of seminal vesicles, this technique is becoming an alternative for the diagnosis and treatment of seminal vesicle and seminal tract diseases $[9,10]$.

However, we have found that there have been issues with low qualification operator-related complications and failures in China recently. Our department used TSV to treat 48 patients from January 2013 to July 2016, performed by 3 surgeons. They were not able to locate the ejaculatory duct opening in some cases, which led to surgical failures; in addition, we have identified other complications, such as epididymitis, urinary tract infection (UTI), ejaculation abnormality (EA), continuous hematuria $(\mathrm{CH})$ and clinical symptoms of rectal injury [11]. The main reason that the low qualification operator training system results in errors is an emphasis on surgical techniques while neglecting the anatomy and holistic view of the seminal tract. We performed a seminal tract anatomical study (STAS), which included the seminal tract and seminal vesicles, studying the structure, morphology, duct trajectory, and anatomical relationships between the seminal vesicles and the adjacent tissues, in pelvic specimens from 12 adult cadavers. Then, we compared the surgical effects and complications between after and before STAS performed by the same 3 surgeons.

\section{Aim}

The aim of this research is to study the guiding role of STAS in TSV.

\section{Material and methods}

\section{Ethical standards}

The present study was approved by the Ethics Committee of Xuzhou Central Hospital and the Medical Ethics Committee of Xuzhou Medical University.

\section{Seminal tract anatomical study}

Twelve pelvic specimens were selected from adult cadavers, and the local structure, morphology, duct trajectory, and anatomical relationships between the seminal vesicles and adjacent tissues were observed. All specimens were provided by the Department of Anatomy, Xuzhou Medical University. The mean age at death was $50.2 \pm 6.4$ years (range: 45-56 years). In all cadavers, the causes of death were not related to prostatic or bladder diseases. No prostate cancer was found in any of the specimens, and the normal structures of the seminal vesicles were not changed.

The entire seminal vesicles and seminal tracts were obtained. Along the routine approach for TSV, the seminal colliculus, prostatic utricle, ejaculatory duct, ejaculatory duct opening, vas deferens ampulla, and seminal vesicles were observed individually, and the length, width, and thickness of the seminal vesicles were measured using a digital micrometer. The seminal colliculus area was observed, and the presence of the prostatic utricle and the length and depth of the prostatic utricle were observed. The observed structures also included the excretory duct of each seminal gland, the trajectory of the vas deferens, the location of the bilateral ejaculatory duct openings, and the trajectory of the ejaculatory duct and its relationship with the prostatic utricle opening. The length and inner diameter of the ejaculatory duct were measured. F3-F6 ureteral catheters were inserted into the bilateral ejaculatory ducts, and the retrograde operation was simulated to observe the morphology, structure, and trajectory of the seminal tract and seminal vesicles and their anatomical relationships with the adjacent tissue.

\section{Surgical procedure of TSV}

Before surgery, transrectal ultrasound and/or magnetic resonance imaging (MRI) were performed to study the prostate, seminal vesicles, and ejaculatory duct. After spinal anesthesia, the patient was placed in the lithotomy position. Then, 4.5-6.5 Fr Storz ureteroscopes were inserted gently and slowly into the posterior urethra through the external urethral orifice to locate the seminal colliculus. Surgeons searched the ejaculatory duct openings at the 5 and 7 o'clock points on the bilateral sides of the prostatic utricle. If necessary, transrectal seminal vesicle massage was performed, and the overflow 
of milky-white fluid helped to identify the ejaculatory duct opening. If the ejaculatory duct opening could not be found, an F3 ureteral catheter was inserted into the prostatic utricle to dilate it slightly, and then the surgeon guided the ureteroscope into the prostatic utricle. After the device entered the prostatic utricle cavity, the surgeon identified the bilateral ejaculatory openings and inserted the ureteroscope into the seminal vesicle through the ejaculatory duct opening under the guidance of the F3 ureteral catheter. If the ejaculatory duct opening was not found, a window was made at the 3-5 o'clock or 7-9 o'clock positions of the bilateral walls of the prostatic utricle to enter the ejaculatory duct. If the prostatic utricle could not be detected, the distal end of the ejaculatory duct was cut open using a plasma urethral resectoscope.

Transurethral resection of the ejaculatory duct was performed by resectoscope plasma electric vaporization. The endoscope was inserted under direct vision, the seminal colliculus, bladder neck, and urethral external sphincter were examined, and both sides of the seminal colliculus were removed carefully. The bilateral ejaculatory ducts were identified observing the overflow of milky-white or yellowish-brown fluid. The endoscope was removed, and TSV was performed. If the ejaculatory duct opening could not be found after these procedures, methylene blue was injected into the vas deferens to identify the ejaculatory duct. The bilateral ampulla of the vas deferens and the internal structure of the seminal vesicle were observed. The presence of congestion, edema, bleeding, seminal vesicle stones, blood clots, neoplasm, or other abnormalities was confirmed by TSV. All the 3 surgeons had performed over 30 cases of TSV respectively before we collected the data to exclude the impact of the learning curve.

\section{Effects and complications study of TSV}

A respectively study was performed to compare the effects and complications after and before the anatomical study. 48 cases of patients who were di- agnosed with obstructive azoospermia and received TSV therapy from January 2013 to July 2016 were enrolled in group $A$. Then, the surgeons attended the anatomical study in August 2016. After that, other 34 cases of patients were enrolled in group B from September 2016 to May 2018.

Baseline information, including age, smoking history, drinking history and body mass index (BMI), was collected and determined preoperatively. Comparisons of the complications included epididymitis, UTI, EA, CH and rectal injury, and the surgical parameters included surgical time (min), catheter days and length of hospital stays (day) before and after the anatomical study.

A total of 82 cases, including 48 patients (group A) and 34 patients (group B) were enrolled in the retrospective study. There were no statistically significant differences in baseline data between groups $A$ and $B$ ( $p>0.05$, Tables I and II).

\section{Statistical analysis}

Statistical analyses were performed using SAS v8.02 software (SAS Institute Inc., Cary, NC, USA). Continuous data are presented as the mean $\pm S D$, and categorical data are presented as a percentage. Continuous data between the two groups were compared using Student's $t$ test. Categorical data were compared using the $\chi^{2}$ test. If $50 \%$ of the cells have expected counts less than 5 , continuity adjustment $\chi^{2}$ test will be performed. A $p<0.05$ was considered significant.

\section{Results}

\section{Results of the anatomical observation}

After the anatomical observation of the seminal tracts and seminal vesicles of 12 adult pelvic specimens, we found the following:

1) The length of the seminal vesicle was $32.6 \pm 6.3 \mathrm{~mm}$ on the left side and $33.1 \pm 5.2 \mathrm{~mm}$ on the right side; the width was $15.7 \pm 3.3 \mathrm{~mm}$ on the left side and $15.2 \pm 4.5 \mathrm{~mm}$ on the right side; and the

Table I. Patient characteristics and preoperative continuous data

\begin{tabular}{|lcccc|}
\hline Parameter & Group A, mean \pm SD & Group B, mean \pm SD & $t$ value & $P$-value \\
\hline Age $[$ years $]$ & $28.0 \pm 3.6$ & $27.9 \pm 4.4$ & 0.15 & 0.8837 \\
\hline BMI $\left[\mathrm{kg} / \mathrm{m}^{2}\right]$ & $26.3 \pm 2.6$ & $26.1 \pm 2.7$ & 0.32 & 0.7510 \\
\hline
\end{tabular}

There were no statistically significant differences in baseline continuous data (all $p>0.05$ ). 
Table II. Patient baseline data

\begin{tabular}{|c|c|c|c|c|}
\hline Parameter & Total, $n$ (\%) & Group A, $n(\%)$ & Group B, $n(\%)$ & $P$-value \\
\hline Patient number & $82(100.0)$ & $48(58.5)$ & 34 (41.5) & \\
\hline Smoking history: & - & - & - & 0.3915 \\
\hline Yes & $31(37.8)$ & $20(24.4)$ & $11(13.4)$ & - \\
\hline No & $51(62.2)$ & $28(34.2)$ & $23(28.1)$ & - \\
\hline Drinking history: & - & - & - & 0.6569 \\
\hline Yes & $22(26.8)$ & $12(14.6)$ & $10(12.2)$ & - \\
\hline No & $60(73.2)$ & $36(43.9)$ & $24(29.3)$ & - \\
\hline Seminal blood: & - & - & - & 0.9736 \\
\hline Yes & $36(43.9)$ & $21(25.6)$ & 15 (18.3) & - \\
\hline No & $46(56.1)$ & 27 (32.9) & $19(23.2)$ & - \\
\hline
\end{tabular}

There were no statistically significant differences in smoking history, drinking history and seminal blood between the 2 groups (all $p>0.05$ ).

thickness was $7.4 \pm 2.1 \mathrm{~mm}$ on the left side and $7.5 \pm 2.8 \mathrm{~mm}$ on the right side.

2) The seminal colliculus was identified in most specimens. The prostatic utricle was observed in 7 of 12 cadavers (58.3\%), while only a tiny depression was observed and the prostatic utricle was not found in the 5 other cases. The mean size of the prostatic utricle opening was 4.8 $\pm 1.4 \mathrm{~mm}$ in the 12 cadavers, and the opening was cave-shaped (Photo 1 A). The prostatic utricle was located between the bilateral ejaculatory ducts. In addition, the interval tissue between two bilateral ejaculatory ducts was thin.

3) The angle between the ejaculatory duct and the midline was $10-15^{\circ}$ at the point where the ejaculatory duct entered the prostate gland (Photo $1 \mathrm{~B})$. The needle-like opening of the ejaculatory duct was found on the seminal colliculus, the size was $0.1 \mathrm{~mm}$, and it was not observed in some specimens. The length of the ejaculatory duct was $14.3 \pm 2.3 \mathrm{~mm}$ on the left side and 14.1 $\pm 3.7 \mathrm{~mm}$ on the right side; the inner diameter was $1.3 \pm 0.4 \mathrm{~mm}$ on the left side and 1.2 $\pm 0.3 \mathrm{~mm}$ on the right side. The ejaculatory duct openings were located at the 5 and 7 o'clock positions on both sides of the prostatic utricle. Following the dilatation of the ejaculatory duct or the seminal vesicle, the ejaculatory duct was extended accordingly (Photo $1 \mathrm{C}$ ).

4) F4-F5 ureteral stents were used in patients with narrow ejaculatory duct openings and for distorted or thin seminal tracts.
5) The lower end of the vas deferens became thin and joined with the duct of the seminal vesicle to form the ejaculatory duct (Photo $1 \mathrm{D})$.

6) The seminal vesicle was honeycomb-shaped, with obvious segmentation in the cross-section, and there was often a liquid residue inside the seminal vesicle (Photo $1 \mathrm{~F}$ ); thus, a full view was never obtained with TSV.

\section{Retrospective study of TSV}

There were no significant differences in the complications between the two groups (all $p>0.05$, Tables III and IV), but the differences in surgical times and surgical validity rates between the two groups were statistically significant ( $p<0.05$, Tables III and IV).

\section{Discussion}

The seminal tract and seminal vesicles are located deep in the body and are hidden. Therefore, traditional surgical treatments for seminal tract and seminal vesicle diseases are relatively complicated, and often the outcomes are not ideal. Jarzemski et al. [5] emphasize that the laparoscopic or robotic technique is an excellent choice for treating seminal vesicle diseases. TSV is a newly developed technique for the diagnosis and treatment of the distal seminal tract and seminal vesicle diseases [12-14]. During the diagnosis and treatment of obstructive azoospermia using TSV, local lesions in the seminal tract and seminal vesicles can be observed, and cysts, stones, and neoplasms in the seminal vesicle 

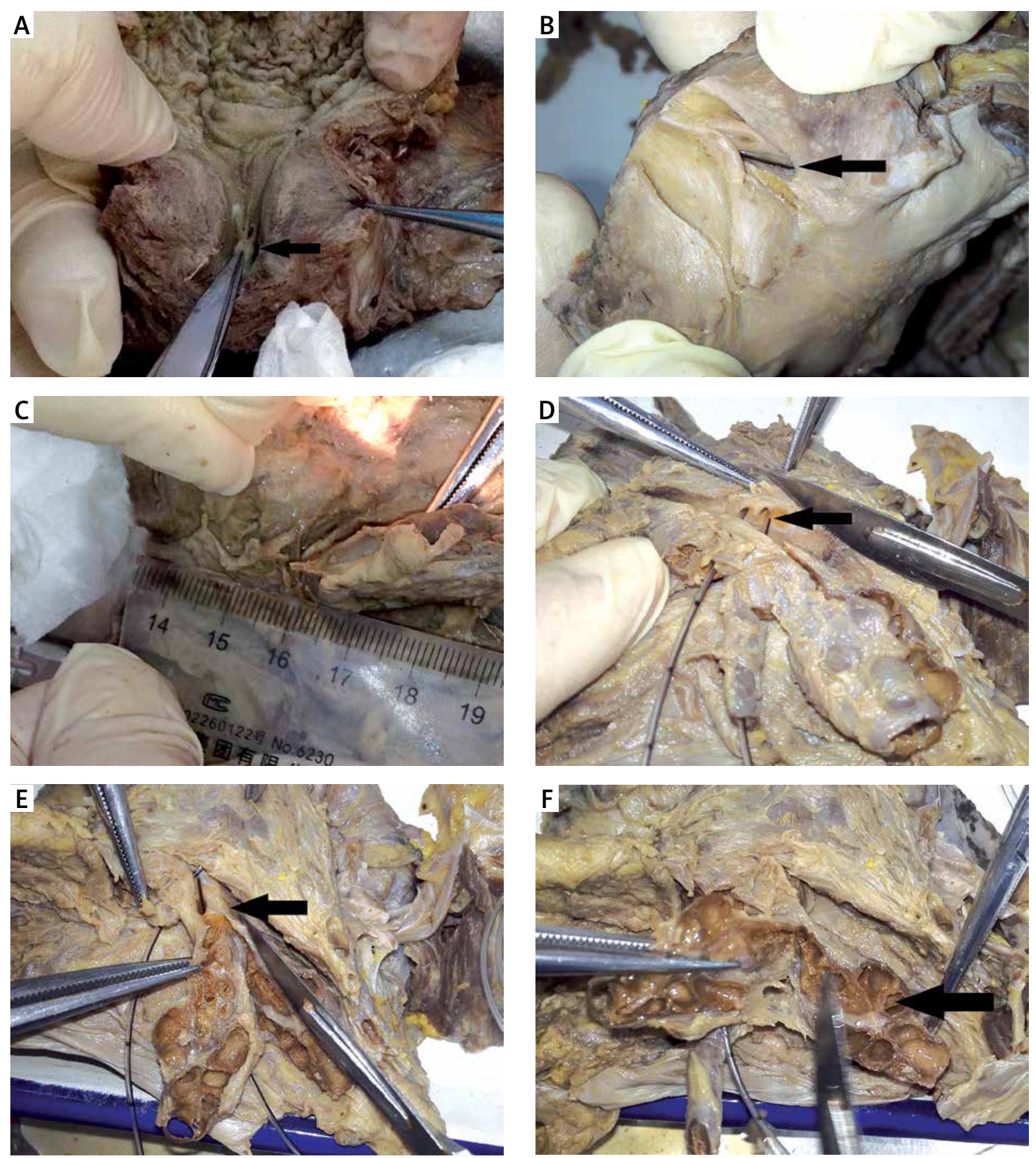

Photo 1. Results of local anatomical study of the specimens. A - The prostatic utricle opening is located at the seminal colliculus of the urethra (black arrow). B - The ejaculatory duct enters the prostate gland (black arrow) and exits from the seminal colliculus. C - The length of the ejaculatory duct is approximately $1.5-3 \mathrm{~cm}$, and it extends in cases with ejaculatory duct dilation or seminal vesicle dilation. D - Bilateral seminal vesicles and the ampulla of the ductus deferens merge into the ejaculatory duct (black arrow) at the dorsal side of the prostate. $\mathbf{E}$ - After dissection, the seminal vesicle is exposed clearly, joins the ampulla of the ductus deferens (black arrow), merges into the ejaculatory duct, and has its opening in the seminal colliculus of the prostatic urethra. F - The seminal vesicle is honeycomb-shaped, with obvious segmentation in the cross-section 
Table III. Surgical parameters

\begin{tabular}{|lcccc|}
\hline Parameter & Group C, mean \pm SD & Group D, mean \pm SD & $t$ value & $P$-value \\
\hline Catheter days & $1.8 \pm 0.7$ & $1.7 \pm 0.7$ & 0.50 & 0.6183 \\
\hline Hospital stays [days] & $4.5 \pm 0.8$ & $4.3 \pm 0.8$ & 1.20 & 0.2325 \\
\hline Surgical time [min] & $24.6 \pm 5.4$ & $21.9 \pm 5.8$ & 2.19 & $0.0311^{\star}$ \\
\hline
\end{tabular}

There were no significant differences in the catheter days and hospital stays between the two groups (both $p>0.05$ ), but the differences in surgical time between the two groups were statistically significant $\left({ }^{*} p<0.05\right)$.

Table IV. Surgical validity rates and complications

\begin{tabular}{|c|c|c|c|c|}
\hline Parameter & Total, $n(\%)$ & Group A, $n(\%)$ & Group B, n (\%) & $P$-value \\
\hline Patient number & $82(100.0)$ & $48(58.5)$ & $34(41.5)$ & \\
\hline Valid: & - & - & - & $0.0236^{*}$ \\
\hline Yes & $59(72.0)$ & $30(36.6)$ & $29(35.4)$ & - \\
\hline No & $23(28.0)$ & $18(22.0)$ & $5(6.1)$ & - \\
\hline Epididymitis: & - & - & - & 0.9877 \\
\hline Yes & $12(14.6)$ & $7(8.5)$ & $5(6.1)$ & - \\
\hline No & $70(85.4)$ & $41(50.0)$ & $29(35.4)$ & - \\
\hline UTI: & - & - & - & $1.0000^{\#}$ \\
\hline Yes & $8(8.8)$ & $5(6.1)$ & $3(3.7)$ & - \\
\hline No & 74 (91.2) & $43(52.4)$ & $31(37.8)$ & - \\
\hline EA: & - & - & - & 0.6509 \\
\hline Yes & $15(18.3)$ & $8(9.8)$ & $7(8.5)$ & \\
\hline No & $67(81.7)$ & $40(48.8)$ & 27 (32.9) & \\
\hline $\mathrm{CH}:$ & - & - & - & 0.5989 \\
\hline Yes & $17(20.7)$ & $9(11.0)$ & $8(9.8)$ & \\
\hline No & 65 (79.3) & 39 (47.6) & $26(31.7)$ & \\
\hline Rectal injury: & - & - & - & $1.0000^{\#}$ \\
\hline Yes & $3(3.7)$ & $2(2.4)$ & $1(1.2)$ & - \\
\hline No & 79 (96.3) & $46(56.1)$ & $33(40.2)$ & - \\
\hline Complications: & - & - & - & 0.7069 \\
\hline Yes & $26(31.7)$ & 16 (19.5) & $10(12.2)$ & \\
\hline No & $56(68.3)$ & $32(39.0)$ & $24(29.3)$ & \\
\hline
\end{tabular}

There were no significant differences in the complications between the two groups (all $p>0.05$ ), but the differences in surgical validity rates between the two groups were statistically significant $\left({ }^{*} p<0.05\right)$. Fifty percentof the cells have expected counts less than 5 , so the continuity adjustment $\chi^{2}$ test was performed $(*)$.

cavity can be treated [15]; therefore, this technique may achieve better outcomes compared with traditional treatments [16]. However, many patients still cannot obtain satisfactory outcomes. Because the seminal tract and seminal vesicle are hidden, thin, and narrow and because no special instruments are available for TSV, the surgery is difficult, as is achieving excellent clinical outcomes. The best method for entering the seminal vesicle safely, accurately, and rapidly during TSV is the most critical issue.
Currently, identifying the location of the ejaculatory duct opening and making an approach for TSV is controversial. Some scholars assert that the ejaculatory duct openings are located at the 5 o'clock and 7 o'clock positions of the seminal colliculus cavity (prostatic utricle) [8, 17, 18]. Wang et al. [19] performed transurethral procedures for 86 patients with benign prostatic hyperplasia or urethral stricture and simultaneously performed transrectal seminal vesicle massage and observed the ejaculatory 
duct openings. They found that two ejaculatory duct openings were located $2 \mathrm{~mm}$ away from the prostatic utricle opening and that these three points formed a triangle or a straight line. Four approaches are recommended for TSV [1]: (1) via the natural opening of the ejaculatory tract; (2) via the prostatic utricle; (3) via the windows made at the 5 or 7 o'clock directions from the prostatic utricle bottom; and (4) via the pathway established by the transurethral resection of the ejaculatory duct opening. However, there are significant differences in validity rates [9, 17-19], which greatly limits the application of this technology. To further improve the success rates and postoperative outcomes of TSV, we selected 12 adult pelvic specimens and studied the structure, morphology, duct trajectory, and anatomical relationships between the seminal vesicles and adjacent tissues.

We identified thin, film-like substances at the 5 and 7 o'clock directions from the prostatic utricle bottom, and entered the seminal vesicle after making a window. Later, we found bilateral ejaculatory openings near the prostatic utricle opening of the seminal colliculus. There were mucosal folds at the opening, which proved to be the ejaculatory duct openings. The seminal colliculus is an eminence at the lower end of the prostatic urethra, and three depressions or orifices can be observed under TSV. The upper depression is the prostatic utricle opening, and the lower two depressions are ejaculatory duct openings. The ejaculatory duct openings are located $2 \mathrm{~mm}$ away from the prostatic utricle opening. In rare pathological situations, the ejaculatory duct opening may be located in the small sac [20].

Through the anatomical observation of the seminal tract, we have improved the validity rate of TSV and shortened the operation time. During surgery, the ejaculatory duct openings can be dilated by Storz F4.5-F6.5 ureteroscopes. After the locations of the ejaculatory ducts are confirmed by semen expulsion from the ejaculatory duct opening, using transrectal massage of the seminal vesicle, the ureteroscope can be inserted via the natural pathway into the seminal vesicle under the guidance of a guidewire. The ureteroscope should be inserted into the seminal vesicle after the dilation of the natural pathway via the ejaculatory duct opening to better protect the surrounding tissue and to avoid seminal tract injury. In addition, these procedures can better protect the peripheral vital tissues and organs, reduce postoperative scar formation, and decrease the recurrence rate. At present, this approach is preferred during TSV in our department.

During the anatomical study of the seminal vesicles and seminal tract in the anatomical observation experiment, we found that the seminal colliculus could be identified clearly in formalin-fixed specimens. The prostatic utricle was observed clearly in 7 of 12 specimens (58.3\%). Morgan et al. [21] reported that the prostatic utricle was observed in 12 out of 13 cases in their series. The prostatic utricle is a dead space between the bilateral ejaculatory ducts and is approximately $6 \mathrm{~mm}$ in length. For quite some time, physicians and anatomical experts have not paid sufficient attention to its structure and function. In our anatomical study, we found that the prostatic utricle was located between the bilateral ejaculatory ducts. There was a thin membrane between the two ejaculatory ducts. We hypothesize that it bulges because of the tension at the time of ejaculatory duct occlusion because it is near the prostatic utricle, and it is the anatomical basis for establishing the ejaculatory duct bypass in the prostatic utricle. Therefore, a window can be made via this area when the natural opening of the ejaculatory duct is not found. However, this is not the preferred approach. When the ejaculatory duct opening is not found, a window should be made at the 5 or 7 o'clock point from the bottom of the prostatic utricle, and this approach is preferred by most surgeons $[22,23]$. However, when we make a hole to enter the ejaculatory duct, it is in theory a false channel; if the size of the hole is too large, it may result in reflux, and if the hole size is too small, it may induce recurrent stenosis or even occlusion after surgery [15].

The outer layer of the ejaculatory duct is longitudinal smooth muscle. At the proximal end, it becomes thin rapidly, and the inner smooth muscle disappears $[8,24]$. Therefore, the ejaculatory duct has no sphincter functions, such as peristalsis or contraction, and urinary reflux is prevented only by the presence of the sharp angle between the ejaculatory duct and the prostatic urethra [23]. An experiment in animals found that the relationship between the seminal vesicle and the ejaculatory duct was similar to that between the bladder and the urethra [25]. When transurethral resection of the ejaculatory duct opening must be performed to enter the ejaculatory duct and the ejaculatory duct is not removed sufficiently, symptoms may still be present after surgery, 
or recurrent stenosis and obstruction may occur; if the ejaculatory duct is removed too extensively, the anti-reflux mechanism may be injured.

According to our anatomical study, the mean length of the ejaculatory duct was $12.2 \pm 3.4 \mathrm{~mm}$, which is consistent with the findings of Nguye et al. [26]. The human ejaculatory duct is $1-2 \mathrm{~cm}$ and gradually becomes thinner from the seminal vesicle to the urethra. The diameters of the proximal, middle, and distal ends of the duct were $0.3-1.74 \mathrm{~mm}$, $0.1-0.64 \mathrm{~mm}$, and only $0.1-0.34 \mathrm{~mm}$, respectively. The ejaculatory duct is generally divided into three segments, the seminal vesicle segment, the prostatic segment, and the seminal colliculus segment. If ejaculatory duct dilation or seminal vesicle dilation occurs, the ejaculatory duct will be extended accordingly. Sometimes, the ejaculatory duct opening is dilated, the ejaculatory duct is extended and enlarged, and a cyst can be formed. At the time of surgery, the ejaculatory duct may be mistaken for the seminal vesicle, which results in misdiagnosis. In the present study, the dilated ejaculatory duct was $1.5 \mathrm{~cm}$ in diameter in one patient, and small yellow granules were present. The dilated ejaculatory duct was initially considered to be the seminal vesicle, and the real seminal vesicle was reached only after repeated manipulations [27, 28].

F3 or F4 ureteral stents can be used for patients with small ejaculatory duct openings or twisted or narrow seminal tracts. Our anatomical study showed that the narrow end of the vas deferens may be located at the junction of the vas deferens and the seminal vesicle, to which sufficient attention has not been paid in the past. The opening we identified was ring-shaped and 1-2 $\mathrm{mm}$ in size. The opening of the vas deferens ampulla is often located on the medial side, and the endoscope may not enter the vas deferens ampulla during TSV. Because the thinnest endoscope at present is still relatively thick for the opening of the vas deferens ampulla, we recommend not entering the proximal end of the vas deferens ampulla to avoid iatrogenic injury and the subsequent obstruction of the vas deferens.

Complication rates in TSV and laparoscopic or robotic techniques were comparable. The incidence of complications of fenestration under seminal vesiculoscopy was $40.0 \%$ (8/20), while the complication rate of transperitoneal laparoscopic unroofing was $36.3 \%$ (12/33) [29]. The complication rate was comparable with the data in our research $(31.7 \%, 26 / 82)$.
Before the operation, the surgeon should fully communicate with the patient, inform the patient of possible surgical outcomes, and allow the patient to consider having his sperm frozen, if necessary, for intracytoplasmic sperm injection. Therefore, we believe that TSV should be performed strictly based on the following surgical indications: (1) the history of intermittent hemospermia should be $>6$ months or $>3$ months, if conservative treatment is ineffective; (2) the imaging examination should suggest anatomic abnormalities, recurrent inflammation, or secondary stone formation in the seminal tract, e.g., an associated ejaculatory duct or seminal vesicle cyst or Mullerian cyst, and the auxiliary examination should suggest ejaculatory duct obstruction or there should be a suspected neoplasm that requires biopsy; and (3) hemospermia caused by prostate cancer or tuberculosis should be excluded.

\section{Conclusions}

According to our experiences with TSV and following our local anatomical study of the seminal tract and seminal vesicles, we believe that minimally invasive transurethral TSV is feasible for the treatment of seminal tract and seminal vesicle diseases. However, as a new surgical procedure, TSV still has some limitations, and better outcomes can be achieved only by combining it with other treatment methods. Our anatomical study of the seminal tract and seminal vesicles has value in guiding TSV in clinical practice. However, our study is still insufficient, due to the limited number of cases and research methods, and thus requires further in-depth clinical and scientific studies. We believe that TSV will have a better future with the development of new minimally invasive endoscopic instruments.

\section{Acknowledgments}

Kun Pang, Wen Yang, Jianjun Zhang and Longjun Cai, Conghui Han and Wenda Zhang contributed equally in this work.

We would like to thank Academician Sun Yinghao and his team from Changhai Hospital for their support of this experiment.

The authors acknowledge financial support from National Natural Science Fund (81774089); Jiangsu Province, the medical innovation team (CXTD-201648); Jiangsu Province key research and development 
program (BE2017635); Jiangsu Provincial Social Development Project (BE2015623); Jiangsu Province, young medical talents (QNRC2016386); Xuzhou Science and Technology Plan Project (KC18036) and Ningbo Natural Science Foundation (2017A610194). The authors acknowledge editorial support from American Journal Experts.

\section{Conflict of interest}

The authors declare no conflict of interest.

\section{References}

1. Lei HE, Zhang XD, Chen R, et al. Transurethral seminal vesiculoscopy for recurrent hemospermia: experience from 419 cases. Andrologia 2018; 20: 438-41.

2. Hu JC, Chen CS. Transurethral seminal vesiculoscopy acts as a therapeutic investigation for intractable hemospermia: stepby-step illustrations and single-surgeon experience. Int J Urol 2018; 25: 589-95.

3. Shah K, Pal B, Rizvi SJ, Modi P. Laparoscopic excision of a congenital seminal vesicle cyst associated with ipsilateral renal agenesis. Urol Int 2011; 87: 238-40.

4. Jang KD, Choi KH, Yang SC, et al. Laparoendoscopic single-site surgery (LESS) for excision of a seminal vesicle cyst associated with ipsilateral renal agenesis. Korean J Urol 2011; 52: 431-3.

5. Jarzemski P, Listopadzki S, Kowalski M. Laparoscopic removal of a congenital seminal vesicle cyst in Zinner's syndrome. JSLS 2014; 18: 367-71.

6. Zhang DX, Li XG, Gao Y, et al. Transperitoneal laparoscopic excision of seminal vesicle cyst: a single-center experience. J Endourol 2012; 26: 1153-8.

7. Schlager D, Maas M, Hein S, et al. Flexible vesiculovasoscopy using a microoptical system in a human cadaver model: an experimental approach for atraumatic endoscopy of the seminal tract. J Endourol 2016; 30: 934-8.

8. Li ZY, Xu Y, Liu C, et al. Anatomical study of the seminal vesicle system for transurethral seminal vesiculoscopy. Clin Anat 2019; 32: 244-52.

9. Han CH, Liang Q, Dong BZ, et al. The transurethral seminal vesiculoscopy in the diagnosis and treatment of the seminal vesicle disease. Cell Biochem Biophys 2013; 66: 851-3.

10. Song T, Zhang X, Zhang L, et al. Transurethral seminal vesiculoscopy in the diagnosis and treatment of seminal vesicle stones. Chin Med J 2012; 125: 1475-8.

11. Tian L, Han H. Clinical features of haematospermia associated with seminal vesicle calculi versus posterior urethral haemangioma. Clin Anat 2018; 50: e13072.

12. Chen R, Wang L, Sheng X, et al. Transurethral seminal vesiculoscopy for recurrent hemospermia: experience from 419 cases. Asian J Androl 2018; 20: 438-41.

13. Zhang W, Xiao G, Qin S, et al. An innovative technique of transurethral seminal vesiculoscopy with ultrasonic lithotripter for severe, persistent hematospermia. J Endourol 2017; 31: 1277-82.

14. Yafi FA. Editorial Comment on: An innovative technique of transurethral seminal vesiculoscopy with ultrasonic litho- tripter for severe, persistent hematospermia by Zhang et al. J Endourology 2017; 31: 1283.

15. Christodoulidou M, Parnham A, Nigam R. Diagnosis and management of symptomatic seminal vesicle calculi. Scand J Urol 2017; 51: 237-44.

16. Cheng G, Liu B, Song Z, et al. A novel surgical management for male infertility secondary to midline prostatic cyst. BMC Urol 2015; 15: 18.

17. Liu C, Song Z, Dun Y, et al. Transurethral electrotomy combined with seminal vesiculoscopy ejaculatory duct dilatation in treating patients with Mullerian duct cyst. Zhong Nan Da Xue Xue Bao Yi Xue Ban 2015; 40: 670-3.

18. Zhu XB, Zhang XS, Zhang SL, et al. 8.5/11.5F transurethral seminal vesiculoscopy in the diagnosis and treatment of refractory hematospermia. Zhonghua Nan Ke Xue 2016; 22: 225-8.

19. Wang L, Liu ZY, Xu CL, et al. Transurethral seminal vesiculoscopy for refractory or recurrent hemospermia: clinical analysis of 162 cases. Zhonghua Nan Ke Xue 2013; 19: 531-4.

20. Xing C, Zhou X, Xin L, et al. Prospective trial comparing transrectal ultrasonography and transurethral seminal vesiculoscopy for persistent hematospermia. Int J Urol 2012; 19: 437-42.

21. Morgan RJ, Williams DI, Pryor JP. Mullerian duct remnants in the male. Br J Urol 1979; 51: 488-92.

22. Liu B, Li J, Li P, et al. Transurethral seminal vesiculoscopy in the diagnosis and treatment of intractable seminal vesiculitis. J Int Med Res 2014; 42: 236-42.

23. Tang SX, Zhou HL, Ding YL. Effectiveness of transurethral seminal vesiculoscopy in the treatment of persistent hematospermia, and oligoasthenozoospermia and azoospermia from ejaculatory duct obstruction. Zhonghua Yi Xue Za Zhi 2016; 96: 2872-5.

24. Shahait M, Averch TD. Editorial Comment on: An innovative technique of transurethral seminal vesiculoscopy with ultrasonic lithotripter for severe persistent hematospermia by Zhang et al. J Endourol 2018; 32: 21.

25. Turek PJ, Aslam K, Younes AK, Nguyen HT. Observations on seminal vesicle dynamics in an in vivo rat model. J Urol 1998; 159: 1731-4.

26. Nguyen HT, Etzell J, Turek PJ. Normal human ejaculatory duct anatomy: a study of cadaveric and surgical specimens. J Urol 1996; 155: 1639-42.

27. Shao GF, Ma TJ, Liu YQ, et al. Real-time transrectal ultrasonography in seminal vesiculoscopy. Zhonghua Nan Ke Xue 2017; 23: 337-42.

28. Li Z, Li XP, Chen HX. Diagnosis and treatment of ejaculatory duct obstruction: current status and advances. Zhonghua Nan Ke Xue 2017; 23: 483-7.

29. Xue RZ, Tang ZY, Chen Z, Huang L. Clinical outcomes of transperitoneal laparoscopic unroofing and fenestration under seminal vesiculoscopy for seminal vesicle cysts. Asian J Androl 2018; 20: 621-5.

Received: 22.03.2019, accepted: 29.05.2019. 